\title{
ON THE UNIFORM PERFECTNESS OF THE BOUNDARY OF MULTIPLY CONNECTED WANDERING DOMAINS
}

\author{
WALTER BERGWEILER ${ }^{\bowtie}$ and JIAN-HUA ZHENG \\ (Received 24 November 2010; accepted 2 September 2011) \\ Communicated by P. C. Fenton
}

\begin{abstract}
We investigate when the boundary of a multiply connected wandering domain of an entire function is uniformly perfect. We give a general criterion implying that it is not uniformly perfect. This criterion applies in particular to examples of multiply connected wandering domains given by Baker. We also provide examples of infinitely connected wandering domains whose boundary is uniformly perfect.
\end{abstract}

2010 Mathematics subject classification: primary 37F10; secondary 30D05.

Keywords and phrases: Fatou set, Julia set, iteration, entire function, wandering domain, uniformly perfect.

\section{Introduction and results}

The Fatou set $F(f)$ of an entire function $f$ is the subset of the complex plane $\mathbb{C}$ where the iterates $f^{n}$ of $f$ form a normal family; that is, every subsequence of $\left(f^{n}\right)$ has a subsequence that converges locally uniformly. Its complement is called the Julia set and denoted by $J(f)$; see [7] for an introduction to and discussion of these sets for transcendental functions.

The connected components of $F(f)$ are called Fatou components. For a Fatou component $U_{0}$ and $k \in \mathbb{N}$, there exists a Fatou component $U_{k}$ containing $f^{k}\left(U_{0}\right)$. A Fatou component $U_{0}$ is called a wandering domain if $U_{j} \neq U_{k}$ when $j \neq k$. While a famous theorem of Sullivan [19] says that rational functions do not have wandering domains, an example of a transcendental entire function with wandering domains had already been constructed before Sullivan's work by Baker [3]. The wandering domains

The first-named author was supported by a Chinese Academy of Sciences Visiting Professorship for Senior International Scientists, Grant No. 2010 TIJ10, the Deutsche Forschungsgemeinschaft, Be 1508/71, the EU Research Training Network CODY and the ESF Networking Programme HCAA. The secondnamed author was supported by Grant No. 10871108 of the NSF of China.

(C) 2011 Australian Mathematical Publishing Association Inc. 1446-7887/2011 \$16.00 
in Baker's example are multiply connected. Baker [2] actually proved that multiply connected Fatou components of transcendental entire functions are always wandering.

Baker's example [3] was the function

$$
f(z)=C z^{2} \prod_{k=1}^{\infty}\left(1+\frac{z}{r_{k}}\right),
$$

where $r_{1}>1$, the constant $C$ is chosen such that $0<C \exp \left(2 / r_{1}\right)<1 / 4$ and $C r_{1}>1$ (for example, $C=1 /(4 e)$ and $r_{1}>4 e$ ), and $\left(r_{k}\right)$ satisfies the recurrence relation

$$
r_{k+1}=C r_{k}^{2} \prod_{j=1}^{k}\left(1+\frac{r_{k}}{r_{j}}\right) .
$$

Then $r_{k+1} \geq 2 r_{k}$ for all $k \in \mathbb{N}$, so the product in (1.1) converges. Baker showed that

$$
f\left(\operatorname{ann}\left(0 ; r_{k}^{2}, \sqrt{r_{k+1}}\right)\right) \subseteq \operatorname{ann}\left(0 ; r_{k+1}^{2}, \sqrt{r_{k+2}}\right)
$$

for large $k$, where $\operatorname{ann}(a ; r, R)=\{z \in \mathbb{C}: r<|z-a|<R\}$; here $0<r<R$ and $a \in \mathbb{C}$. This implies that

$$
\operatorname{ann}\left(0 ; r_{k}^{2}, \sqrt{r_{k+1}}\right) \subseteq U_{k}
$$

for some multiply connected Fatou component $U_{k}$. In fact, Baker had constructed the example and verified these properties much earlier [1], but in that paper the question whether the $U_{k}$ are all different had remained open. It was only in [3] that he could prove that all the $U_{k}$ are different and thus wandering domains.

Many properties of this example are typical for functions with multiply connected Fatou components. We collect some of these properties in the following theorem. Here $n(\gamma, a)$ denotes the winding number of a curve $\gamma$ with respect to the point $a$.

THEOREM 1.1. Let $f$ be a transcendental entire function with a multiply connected wandering domain $U_{0}$ and, for $k \in \mathbb{N}$, let $U_{k}$ be the component of $F(f)$ containing $f^{k}(U)$. Then the following properties hold.

(i) $\left.f^{k}\right|_{U_{0}} \rightarrow \infty$ as $k \rightarrow \infty$.

(ii) Each $U_{k}$ contains a closed curve $\gamma_{k}$ satisfying $n\left(\gamma_{k}, 0\right) \neq 0$ for large $k$; in fact, if $\gamma_{0}$ is a Jordan curve in $U_{0}$ that is not null-homotopic, then we may take $\gamma_{k}=f^{k}\left(\gamma_{0}\right)$.

(iii) Each $U_{k}$ is bounded.

(iv) Each $U_{k}$ is contained in a bounded component of the complement of $U_{k+1}$ for large $k$.

(v) There exist sequences $\left(r_{k}\right)$ and $\left(R_{k}\right)$ tending to $\infty$ such that

$$
\operatorname{ann}\left(0 ; r_{k}, R_{k}\right) \subseteq U_{k}
$$

for large $k$ and $\lim _{k \rightarrow \infty} R_{k} / r_{k}=\infty$. 
Here (i) is a simple observation apparently first made by Töpfer [20, p. 67], and (ii) and (iii) are due to Baker [4, Theorem 3.1] (he states only the first part of (ii), but his proof gives the second part too). Next, (iv) is an easy consequence of (ii). Finally, (v) was proved in [23].

Baker [5, Theorem 2] modified his construction to show that there exists an entire function $f$ of the form

$$
f(z)=C^{2} \prod_{k=1}^{\infty}\left(1+\frac{z}{r_{k}}\right)^{2}
$$

with a multiply connected Fatou component of infinite connectivity; see Section 6. In the opposite direction, Kisaka and Shishikura [12] constructed examples where the connectivity is finite, thereby answering a question of Baker. In fact, they showed that for every $N \in \mathbb{N}$ there exists an entire function with an $N$-connected Fatou component.

Next we recall that a closed subset $K$ of $\mathbb{C}$ is called uniformly perfect if there exists $c \in(0,1)$ such that $\operatorname{ann}(a ; c r, r) \cap K \neq \emptyset$ if $a \in K$ and $0<r<\operatorname{diam}(K)$. An equivalent condition is that the modulus $\bmod (A)$ of any annulus $A$ separating two components of $K$ is bounded (that is, $\bmod (A) \leq C$ for some $C$ ). Here by an annulus we mean a doubly connected domain and the modulus of an annulus $A$ is given by

$$
\bmod (A)=\frac{1}{2 \pi} \log \frac{R}{r},
$$

where $r$ and $R$ are such that $A$ can be mapped conformally onto ann $(0 ; r, R)$; the ratio $R / r$ does not depend on the conformal map. The concept of uniform perfectness was introduced by Beardon and Pommerenke $[6,15]$ and has found many applications in complex analysis.

It was proved by Mañé and da Rocha [13], Hinkkanen [11] and Eremenko [10], with different proofs, that Julia sets of rational functions are uniformly perfect. However, the sequence $\left(r_{k}\right)$ in Baker's example (1.1) satisfies $\lim _{k \rightarrow \infty} \sqrt{r_{k+1}} / r_{k}^{2}=\infty$ and this implies that $J(f)$ is not uniformly perfect for this function $f$. In fact, it follows from Theorem $1.1(\mathrm{v})$ that the Julia set of an entire function with a multiply connected Fatou component is never uniformly perfect.

Here we study the question of when the boundary of a multiply connected Fatou component is uniformly perfect. Clearly, this is the case for Fatou components of finite connectivity, so it suffices to consider infinitely connected Fatou components.

Denote by $\widehat{\mathbb{C}}$ the one-point compactification of $\mathbb{C}$. Given a domain $U$ in $\mathbb{C}$ and $a \in \widehat{\mathbb{C}} \backslash U$, we denote by $C(a, U)$ the component of $\widehat{\mathbb{C}} \backslash U$ that contains $a$, and we put $C(a, U)=\emptyset$ if $a \in U$. The union of $U$ and its bounded complementary components is denoted by $\widetilde{U}$. Thus $\widetilde{U}=\mathbb{C} \backslash C(\infty, U)$. The set of critical points of $f$ is denoted by $\operatorname{crit}(f)$.

THEOREM 1.2. Let $f$ be a transcendental entire function with a multiply connected Fatou component $U_{0}$ and put $U_{k}=f^{k}\left(U_{0}\right)$ for $k \in \mathbb{N}$. Denote by $l_{k}$ the number of 
critical points $c$ of $f$ in $\widetilde{U}_{k}$ for which $f(c) \notin C\left(0, U_{k+1}\right)$, by $m_{k}$ the number of zeros of $f$ in $C\left(0, U_{k}\right)$, and by $n_{k}$ the number of zeros of $f$ in $\widetilde{U}_{k}$. Suppose that

$$
l_{k}<m_{k}
$$

for all large $k$. Suppose also that there exist infinitely many positive integers $k$ such that $U_{k} \cap \operatorname{crit}(f) \neq \emptyset$ and $U_{k+1}$ contains an annulus $A_{k+1}$ that separates $U_{k+1} \cap f(\operatorname{crit}(f))$ from $C\left(0, U_{k+1}\right)$, and that

$$
\frac{\bmod \left(A_{k+1}\right)}{n_{k}-m_{k}} \rightarrow \infty .
$$

Then $\partial U_{0}$ is not uniformly perfect.

It is not hard to see that $f$ has $n_{k}-m_{k}$ critical points in $\widetilde{U}_{k} \backslash C\left(0, U_{k}\right)$. In particular, $n_{k}>m_{k}$ if $U_{k}$ contains a critical point. Thus the denominator in (1.5) is nonzero. Note that $l_{k} \leq n_{k}-m_{k}$, so that (1.4) is satisfied if

$$
n_{k}<2 m_{k}
$$

We note that $n_{k}-m_{k}$ and hence $l_{k}$ are bounded in the examples (1.1) and (1.3). Thus (1.4) and (1.6) are satisfied there.

While the hypotheses of Theorem 1.2 may seem somewhat complicated, the result works well for specific examples. We use Theorem 1.2 to show that in Baker's original example of a multiply connected wandering domain, that is, the example given by (1.1) and (1.2), the connectivity is infinite and the boundary is not uniformly perfect. The question whether the connectivity of this domain is finite or infinite was raised by Baker [5] and by Kisaka and Shishikura [12]; the question was repeated in [8, p. 2946] and [16, p. 312].

More generally, we give a fairly complete discussion of functions of order zero where the moduli $r_{k}$ of the zeros satisfy a recursion formula similar to (1.2), with initial values chosen such that $r_{k+1} \geq 2 r_{k}$ for large $k$. Our result also shows that an infinitely connected wandering domain may have a uniformly perfect boundary.

THEOREM 1.3. Let $\left(r_{k}\right)$ and $\left(P_{k}\right)$ be sequences of positive numbers satisfying $r_{k+1} \geq 2 r_{k}$ for large $k$,

$$
\lim _{k \rightarrow \infty} \sqrt[k]{P_{k}}=1
$$

and

$$
r_{k+1}=P_{k} r_{k}^{N} \prod_{j=1}^{k}\left(1+\frac{r_{k}}{r_{j}}\right)
$$

for some nonnegative integer $N$ and all $k$. Let $C \in \mathbb{C} \backslash\{0\}$, let $\left(a_{k}\right)$ be a sequence of complex numbers satisfying $\left|a_{k}\right|=r_{k}$, and define the entire function $f$ by

$$
f(z)=C z^{N} \prod_{k=1}^{\infty}\left(1-\frac{z}{a_{k}}\right) .
$$


Then there exist $K \in \mathbb{N}$ and a sequence $\left(\varepsilon_{k}\right)$ of positive real numbers tending to 0 such that

$$
f\left(\operatorname{ann}\left(0 ;\left(1+\varepsilon_{k}\right) r_{k},\left(1-\varepsilon_{k}\right) r_{k+1}\right)\right) \subseteq \operatorname{ann}\left(0 ;\left(1+\varepsilon_{k+1}\right) r_{k+1},\left(1-\varepsilon_{k+1}\right) r_{k+2}\right)
$$

when $k \geq K$.

Denote, when $k \geq K$, the Fatou component containing

$$
\operatorname{ann}\left(0 ;\left(1+\varepsilon_{k}\right) r_{k},\left(1-\varepsilon_{k}\right) r_{k+1}\right)
$$

by $U_{k}$. If

$$
\limsup _{k \rightarrow \infty} k P_{k}>\frac{|C|}{2 e} \quad \text { or } \quad \liminf _{k \rightarrow \infty} k P_{k}<\frac{|C|}{2 e},
$$

then $U_{k}$ is infinitely connected for all $k$, and if

$$
\limsup _{k \rightarrow \infty} k P_{k}>\frac{|C|}{2 e} \quad \text { or } \quad \liminf _{k \rightarrow \infty} k P_{k}=0,
$$

then $\partial U_{k}$ is not uniformly perfect for all $k$. If

$$
\limsup _{k \rightarrow \infty} k P_{k}<\frac{|C|}{2 e} \text { and } \quad \liminf _{k \rightarrow \infty} k P_{k}>0,
$$

then $\partial U_{k}$ is uniformly perfect for all $k$.

Noting that $P_{k}=C$ and thus $\lim _{k \rightarrow \infty} k P_{k}=\infty$ in Baker's first example of a wandering domain, we deduce that this wandering domain is infinitely connected and that its boundary is not uniformly perfect.

In principle, a similar discussion would be possible for functions of the form

$$
f(z)=C z^{N} \prod_{k=1}^{\infty}\left(1-\frac{z}{a_{k}}\right)^{2},
$$

but here we will only prove that in Baker's example (1.3) of an infinitely connected wandering domain the boundary is also not uniformly perfect; see Section 6.

In the functions considered in Theorem 1.3, as well as in Baker's example (1.3), $n_{k}-m_{k} \leq 2$ and thus the condition (1.5) just says that $\bmod \left(A_{k+1}\right) \rightarrow \infty$. In the next example, $n_{k}-m_{k} \rightarrow \infty$. This shows that (1.5) is best possible in some sense.

THEOREM 1.4. Let $q_{0}$ be an even integer and put $a_{0}=\exp \left(q_{0} / 2\right)$. Define sequences $\left(q_{k}\right)$ and $\left(a_{k}\right)$ recursively by

$$
q_{k+1}=\frac{3}{2} q_{k}^{2} \quad \text { and } \quad a_{k+1}=\exp q_{k}
$$

and the function $f$ by

$$
f(z)=z^{2} \prod_{k=0}^{\infty}\left(1-\frac{z}{a_{k}}\right)^{q_{k}} .
$$


The function $f$ is entire, and if $q_{0}$ is sufficiently large, then $f$ has an infinitely connected Fatou component $U_{0}$ whose boundary is uniformly perfect.

Moreover, (1.4) is satisfied, each $U_{k}$ contains exactly one critical point $c_{k}$ and $U_{k+1}$ contains an annulus $A_{k+1}$ separating $f\left(c_{k}\right)$ from $C\left(0, U_{k+1}\right)$ such that

$$
\frac{\bmod \left(A_{k+1}\right)}{n_{k}-m_{k}} \geq c
$$

for some positive constant $c$ and all $k$.

There is an interesting difference between the example given by Theorem 1.4 and the examples obtained from Theorem 1.3 by choosing $\left(P_{k}\right)$ such that $(1.10)$ holds. Our proofs will show that the complementary components of the wandering domain in the example of Theorem 1.4 cluster only at the 'outer boundary', while they cluster at the 'inner boundary' in the examples obtained from Theorem 1.3. We discuss this in more detail at the end of Section 5.

\section{Preliminaries}

We denote the connectivity of a domain $G$ in $\mathbb{C}$ by $\operatorname{conn}(G)$; that is, $\operatorname{conn}(G)$ is the number of connected components of $\widehat{\mathbb{C}} \backslash G$. The following result is known as the Riemann-Hurwitz formula; see, for example, [17, p. 7].

Lemma 2.1. Let $G$ and $H$ be domains in $\mathbb{C}$ and let $f: G \rightarrow H$ be a proper holomorphic map of degree $d$ with $m$ critical points, counting multiplicity. Then

$$
\operatorname{conn}(G)-2=d(\operatorname{conn}(H)-2)+m
$$

In particular, if one of the domains is of infinite connectivity, then so is the other.

It follows from Theorem 1.1 (iii) that if $f$ is an entire function with a multiply connected wandering domain $U_{0}$ and $U_{k}$ is the component of $F(f)$ containing $f^{k}(U)$, then $f: U_{k} \rightarrow U_{k+1}$ is a proper map. In particular, $U_{k}=f^{k}\left(U_{0}\right)$.

The following consequence of the Riemann-Hurwitz formula may be found in [5, Lemma 6] and [12, Theorem A].

LEMMA 2.2. Let $f$ be a transcendental entire function with a multiply connected wandering domain $U_{0}$. If $\bigcup_{k=0}^{\infty} f^{k}(U)$ contains infinitely many critical points, then $U_{0}$ is infinitely connected.

It was shown in [9, Theorem 1.7] that the converse also holds: if $\bigcup_{k=0}^{\infty} U_{k}$ contains only finitely many critical points, then $U_{0}$ has finite connectivity. We will not need this result, but we mention that it shows that the hypothesis in Theorem 1.2 that infinitely many $U_{k}$ contain a critical point is automatically fulfilled if $U_{0}$ is infinitely connected. 
Lemma 2.3. Let $G$ and $H$ be simply-connected domains, $f: G \rightarrow H$ be a proper holomorphic map of degree $d$, and $A$ be an annulus in $H$. Denote by $p$ the number of critical points $c \in G$ for which $f(c) \in \widetilde{A}$, counted according to multiplicity. If $d>2 p$, then $f^{-1}(A)$ has a component $B$ such that $f: B \rightarrow A$ is univalent. In particular, $\bmod (B)=\bmod (A)$.

Proof. Let $C_{1}, \ldots, C_{k}$ be the components of $f^{-1}(\widetilde{A})$ and $r_{j}$ be the number of critical points in $C_{j}$. Then $\sum_{j=1}^{k} r_{j}=p$. Now $f: C_{j} \rightarrow \widetilde{A}$ is a proper map, whose degree is $r_{j}+1$ by the Riemann-Hurwitz formula, since $C_{j}$ and $\widetilde{A}$ are simply connected. Thus

$$
d=\sum_{j=1}^{k}\left(r_{j}+1\right)=k+\sum_{j=1}^{k} r_{j}=k+p .
$$

By hypothesis, $d>2 p$, and this implies that $k>p$. Thus there exists $j$ such that $C_{j}$ contains no critical point. Hence $f: C_{j} \rightarrow \widetilde{A}$ is univalent and the conclusion follows.

We denote the density of the hyperbolic metric in a hyperbolic domain $U$ by $\varrho_{U}$ and the hyperbolic length of a curve $\gamma$ in $U$ by length $(\gamma, U)$. Thus

$$
\text { length }(\gamma, U)=\int_{\gamma} \varrho_{U}(z)|d z| \text {. }
$$

The following result is well known; see [18, Theorem 2.3] or [22, Proposition 3].

Lemma 2.4. Let $U$ be a hyperbolic domain. Then $\partial U$ is uniformly perfect if and only if there exists a positive $\delta$ such that length $(\gamma, U) \geq \delta$ for each curve $\gamma$ in $U$ that is not null-homotopic.

The next lemma is also standard, but for convenience we include the proof. Here $n(\gamma, a)$ denotes the winding number of a curve $\gamma$ with respect to a point $a$.

Lemma 2.5. Let $0<r<R$ and let $\gamma$ be a closed curve in ann $(0 ; r, R)$. Then

$$
\operatorname{length}(\gamma, \operatorname{ann}(0 ; r, R)) \geq \frac{2 \pi^{2}|n(\gamma, 0)|}{\log (R / r)}
$$

Proof. We may assume that $r=1$. The density of the hyperbolic metric in $\operatorname{ann}(0 ; 1, R)$ is given by (see, for example, [14, p. 12])

$$
\varrho_{\text {ann }(0 ; 1, R)}(z)=\frac{\pi}{|z| \sin (\pi \log |z| / \log R) \log R} .
$$

In particular,

$$
\varrho_{\text {ann }(0 ; 1, R)}(z) \geq \frac{\pi}{|z| \log R}
$$


and thus

$$
\begin{aligned}
\operatorname{length}(\gamma, \operatorname{ann}(0 ; 1, R)) & =\int_{\gamma} \varrho_{\operatorname{ann}(0 ; 1, R)}(z)|d z| \\
& \geq \frac{\pi}{\log R} \int_{\gamma} \frac{|d z|}{|z|} \\
& \geq \frac{\pi}{\log R} \int_{\gamma}|d(\arg z)| \\
& \geq \frac{\pi}{\log R}\left|\int_{\gamma} d(\arg z)\right| \\
& =\frac{2 \pi^{2}}{\log R}|n(\gamma, 0)|,
\end{aligned}
$$

as required.

The following result may be found in [21, Theorem 3].

Lemma 2.6. Let $U$ and $V$ be domains in $\mathbb{C}$ and let $f: U \rightarrow V$ be a proper holomorphic map. Then $\partial U$ is uniformly perfect if and only if $\partial V$ is uniformly perfect.

Lemma 2.6 implies that if an entire function $f$ has a multiply connected wandering domain $U_{0}$ and $U_{k}$ is the Fatou component containing $f^{k}(U)$, so that $U_{k}=f^{k}(U)$ as remarked after Lemma 2.1, then $\partial U_{0}$ is uniformly perfect if and only if $\partial U_{k}$ is uniformly perfect.

\section{Proof of Theorem 1.2}

It follows from Theorem 1.1 (ii) that

$$
0 \notin U_{k} \quad \text { and } \quad 0 \in \widetilde{U}_{k}
$$

for large $k$. In view of Lemma 2.6 and the following remark, we may assume that (3.1) holds for all $k \geq 0$.

Now let $k$ be an index such that $U_{k}$ contains a critical point $c_{k}$ and let $A_{k+1}$ be an annulus as given in the hypothesis. Choosing $A_{k+1}$ slightly smaller if necessary, we may assume that $f(c) \notin \bar{A}_{k+1}$ for every critical point $c \in U_{k}$ and that $\bar{A}_{k+1} \subset U_{k+1}$. Thus there exists an annulus $B_{k+1} \subset U_{k+1}$ separating $A_{k+1}$ and $C\left(\infty, U_{k+1}\right)$ such that $f\left(c_{k}\right) \in B_{k+1}$. We may also assume that $A_{k+1}$ and $B_{k+1}$ are bounded by smooth curves.

Let $V_{k}$ be the component of $f^{-1}\left(B_{k+1}\right) \cap U_{k}$ that contains $c_{k}$. By the RiemannHurwitz formula, $V_{k}$ is at least triply connected. Thus there exists a component $X_{k}$ of $\widehat{\mathbb{C}} \backslash V_{k}$ satisfying $X_{k} \neq C\left(0, V_{k}\right)$ and $X_{k} \neq C\left(\infty, V_{k}\right)$. Hence $0 \notin X_{k}$ and $X_{k} \subset \widetilde{U}_{k}$. Moreover, $X_{k} \cap U_{k}$ contains a component $Y_{k}$ of $f^{-1}\left(A_{k+1}\right)$. Since $f(c) \notin A_{k+1}$ for every critical point $c \in U_{k}$, we find that $Y_{k}$ is also an annulus and $f: Y_{k} \rightarrow A_{k+1}$ is a covering map. Moreover, $f: \widetilde{Y}_{k} \rightarrow \widetilde{A}_{k+1}$ is a proper map and its degree $d_{k}$ is equal to the number 
of zeros of $f$ in $\widetilde{Y}_{k}$. Now $\widetilde{Y}_{k} \subset X_{k} \subset \widetilde{U}_{k} \backslash C\left(0, U_{k}\right)$, so $d_{k} \leq n_{k}-m_{k}$. On the other hand, $d_{k}$ is equal to the degree of the covering $f: Y_{k} \rightarrow A_{k+1}$. Thus

$$
\bmod \left(Y_{k}\right)=\frac{\bmod \left(A_{k+1}\right)}{d_{k}} \geq \frac{\bmod \left(A_{k+1}\right)}{n_{k}-m_{k}} .
$$

Hence $U_{k}$ contains an annulus $Y_{k}$ such that $0 \notin \widetilde{Y}_{k}$ and $\bmod \left(Y_{k}\right) \rightarrow \infty$ as $k \rightarrow \infty$.

Now let $p_{k-1}$ be the number of critical points $c$ of $f$ in $\widetilde{U}_{k-1}$ for which $f(c) \in \widetilde{Y}_{k}$. Since $\widetilde{Y}_{k} \subset X_{k}$ and $X_{k} \neq C\left(0, U_{k}\right)$, we have $p_{k-1} \leq l_{k-1}$. Without loss of generality, we may assume that (1.4) holds for all $k$ and thus $p_{k-1}<m_{k-1}$. Since $n_{k-1}$ is the degree of the proper map $f: \widetilde{U}_{k-1} \rightarrow \widetilde{U}_{k}$, we have $n_{k-1} \geq m_{k-1}+l_{k-1}>2 p_{k-1}$. We deduce from Lemma 2.3 that there exists an annulus $Y_{k}^{1} \subset U_{k-1}$ that is mapped univalently onto $Y_{k}$ by $f$. Applying Lemma 2.3 again, we find an annulus $Y_{k}^{2} \subset U_{k-2}$ that is mapped univalently onto $Y_{k}$ by $f^{2}$. Inductively, we thus obtain a nontrivial annulus $Y_{k}^{k} \subset U_{0}$ that is mapped univalently onto $Y_{k}$ by $f^{k}$. Since $\bmod \left(Y_{k}^{k}\right)=\bmod \left(Y_{k}\right) \rightarrow \infty$ as $k \rightarrow \infty$ by our hypothesis $(1.5)$, we conclude that $\partial U_{0}$ is not uniformly perfect.

\section{Proof of Theorem 1.3}

Since $r_{k+1} \geq 2 r_{k}$ for large $k$, we see easily that the product defining $f$ converges. Now

$$
r_{k+1}=P_{k} r_{k}^{N} \prod_{j=1}^{k}\left(1+\frac{r_{k}}{r_{j}}\right) \geq P_{k} \frac{r_{k}}{r_{1}} \prod_{j=2}^{k-1} \frac{r_{k}}{r_{j}} \geq P_{k} \frac{r_{k}}{r_{1}}\left(\frac{r_{k}}{r_{k-1}}\right)^{k-2}
$$

when $k \geq 2$. Together with (1.7), this implies not only that $r_{k+1} \geq 2 r_{k}$, but also that

$$
\lim _{k \rightarrow \infty} \frac{r_{k+1}}{r_{k}}=\infty \text {. }
$$

Moreover,

$$
\frac{r_{k+1}}{r_{k}} \geq \frac{P_{k}}{r_{1}}\left(\frac{r_{k}}{r_{k-1}}\right)^{k-2} \geq \frac{P_{k}}{r_{1}} 3^{k-k_{0}} \geq 2^{k}
$$

for some $k_{0}$ and large $k \geq k_{0}$. This implies that if $w \in \mathbb{C} \backslash\{0\}$, then

$$
\left|\prod_{j=1}^{k-1}\left(1-\frac{w a_{k}}{a_{j}}\right)\right| \sim\left|w^{k-1} \prod_{j=1}^{k-1} \frac{a_{k}}{a_{j}}\right|=|w|^{k-1} \prod_{j=1}^{k-1} \frac{r_{k}}{r_{j}} \sim|w|^{k-1} \prod_{j=1}^{k-1}\left(1+\frac{r_{k}}{r_{j}}\right)
$$

and

$$
\left|\prod_{j=k+1}^{\infty}\left(1-\frac{w a_{k}}{a_{j}}\right)\right| \rightarrow 1
$$


as $k \rightarrow \infty$. Given $\beta>\alpha>0$, we actually find that (4.2) and (4.3) hold uniformly for $w$ such that $\alpha \leq|w| \leq \beta$. It follows that

$$
\begin{aligned}
\left|f\left(w a_{k}\right)\right| & \sim\left|C\left(w a_{k}\right)^{N}\right||w|^{k-1} \prod_{j=1}^{k-1}\left(1+\frac{r_{k}}{r_{j}}\right)|w-1| \\
& =|C||w|^{k-1+N} r_{k}^{N} \prod_{j=1}^{k-1}\left(1+\frac{r_{k}}{r_{j}}\right)|w-1| \\
& =|C| \frac{|w|^{k-1+N}|w-1|}{2 P_{k}} r_{k+1}
\end{aligned}
$$

as $k \rightarrow \infty$, uniformly for these $w$.

Fix $\varepsilon \in(0,1 / 3]$. We deduce from (4.4) that if $|z|=(1+\varepsilon) r_{k}=(1+\varepsilon)\left|a_{k}\right|$, then

$$
|f(z)| \geq(1-o(1)) \frac{|C|(1+\varepsilon)^{k-1+N} \varepsilon}{2 P_{k}} r_{k+1}
$$

and thus

$$
|f(z)| \geq 2 r_{k+1}
$$

if $k$ is sufficiently large. Similarly we find that

$$
|f(z)| \leq \frac{1}{2} r_{k+1}
$$

when $|z|=(1-\varepsilon) r_{k}$ and $k$ is large. Moreover, it follows from this reasoning and (4.1) that if $|z|=(1-\varepsilon) r_{k}$, then

$$
\begin{aligned}
|f(z)| & \geq(1-o(1)) \frac{|C|(1-\varepsilon)^{k-1+N} \varepsilon}{2 P_{k}} r_{k+1} \\
& \geq(1-o(1)) \frac{|C|(1-\varepsilon)^{k-1+N} \varepsilon 2^{k}}{2 P_{k}} r_{k} .
\end{aligned}
$$

Thus

$$
|f(z)| \geq 2 r_{k}
$$

when $|z|=(1-\varepsilon) r_{k}$ for large $k$.

Finally, replacing $k$ by $k+1$ in (4.6), we conclude that $|f(z)| \leq r_{k+2} / 2$ when $|z|=$ $(1-\varepsilon) r_{k+1}$. Now $(1-\varepsilon) r_{k+1} \geq(1+\varepsilon) r_{k}$, since $r_{k+1} \geq 2 r_{k}$ and $0<\varepsilon \leq 1 / 3$. Thus, by the maximum principle, the last inequality also holds when $|z|=(1+\varepsilon) r_{k}$. Hence

$$
|f(z)| \leq \frac{1}{2} r_{k+2}
$$

when $|z|=(1+\varepsilon) r_{k}$. Now (1.9) follows from (4.5)-(4.8) and the maximum and minimum principle. 
Next we show that, for large $k$, the function $f$ has exactly one critical point, $c_{k}$ say, in the closed annulus $B_{k}=\overline{\operatorname{ann}}\left(0 ; \sqrt{r_{k} r_{k-1}}, \sqrt{r_{k+1} r_{k}}\right)$, and

$$
c_{k}=\left(1-\frac{1}{k+N+\delta_{k}}\right) a_{k}
$$

for some sequence $\left(\delta_{k}\right)$ that tends to 0 . In order to do this, we note that if $z \in B_{k}$, then

$$
\begin{aligned}
\mid \frac{f^{\prime}(z)}{f(z)} & -\frac{k-1+N}{z}-\frac{1}{z-a_{k}} \mid \\
& =\left|\sum_{j=1}^{k-1}\left(\frac{1}{z-a_{j}}-\frac{1}{z}\right)+\sum_{j=k+1}^{\infty} \frac{1}{z-a_{j}}\right| \\
& \leq \sum_{j=1}^{k-1} \frac{r_{j}}{|z|\left(|z|-r_{j}\right)}+\sum_{j=k+1}^{\infty} \frac{1}{r_{j}-|z|} \leq \sum_{j=1}^{k-1} \frac{2 r_{j}}{|z|^{2}}+\sum_{j=k+1}^{\infty} \frac{2}{r_{j}} \\
& \leq \frac{4 r_{k-1}}{|z|^{2}}+\frac{4}{r_{k+1}} \leq 4\left(\sqrt{\frac{r_{k-1}}{r_{k}}}+\sqrt{\frac{r_{k}}{r_{k+1}}}\right) \frac{1}{|z|}=o\left(\frac{1}{|z|}\right)
\end{aligned}
$$

as $k \rightarrow \infty$.

Using Rouché's theorem, we deduce from (4.10) that the difference between the number of zeros and poles in $B_{k}$ is the same for $f^{\prime} / f$ and the function given by

$$
z \mapsto \frac{k-1+N}{z}+\frac{1}{z-a_{k}},
$$

provided $k$ is sufficiently large. We conclude that if $k$ is large, then $f^{\prime}$ has exactly one zero, $c_{k}$ say, in $B_{k}$. Moreover, (4.10) implies that

$$
\frac{k-1+N}{c_{k}}+\frac{1}{c_{k}-a_{k}}=o\left(\frac{1}{\left|c_{k}\right|}\right)
$$

as $k \rightarrow \infty$. This yields (4.9).

It follows from (4.4) and (4.9) that

$$
\left|f\left(c_{k}\right)\right| \sim \frac{|C|}{2 P_{k}}\left|1-\frac{1}{k+N+\delta_{k}}\right|^{k-1+N}\left|\frac{1}{k+N+\delta_{k}}\right| r_{k+1} \sim \frac{|C|}{2 e k P_{k}} r_{k+1}
$$

as $k \rightarrow \infty$.

Suppose now that $\lim \sup _{k \rightarrow \infty} k P_{k}>|C| /(2 e)$. Then there exists $\varepsilon>0$ such that

$$
\left|f\left(c_{k}\right)\right|<(1-\varepsilon) r_{k+1}
$$

for infinitely many $k$. However,

$$
\left|f\left(c_{k}\right)\right|>(1-\varepsilon) \frac{|C|}{2 e k P_{k}} r_{k+1} \geq(1-\varepsilon) \frac{|C| 3^{k-k_{0}}}{2 e k r_{1}} r_{k} \geq k r_{k}
$$


for large $k$ by (4.1) and (4.11). Thus $f\left(c_{k}\right) \in U_{k}$ for infinitely many $k$ and Lemma 2.2 implies that all $U_{k}$ are infinitely connected.

Moreover, noting that $n_{k-1}-m_{k-1} \leq 2$, we see that (1.5), with $k$ replaced by $k-1$, holds for

$$
A_{k}=\operatorname{ann}\left(0 ;\left(1+\varepsilon_{k}\right) r_{k}, k r_{k}\right) \subseteq U_{k} .
$$

Clearly, (1.6) and hence (1.4) are also satisfied. Theorem 1.2 shows that $\partial U_{k}$ is not uniformly perfect if $k \geq K$.

Similarly, if $\lim _{\inf _{k \rightarrow \infty}} k P_{k}<|C| /(2 e)$, then

$$
(1+\varepsilon) r_{k+1}<\left|f\left(c_{k}\right)\right|<2^{k} r_{k+1} \leq \frac{1}{2} r_{k+2}
$$

and hence $f\left(c_{k}\right) \in U_{k+1}$ for infinitely many $k$ by (1.7), (4.1) and (4.11). Again from Lemma 2.2, all $U_{k}$ are infinitely connected. Moreover, if $\lim _{\inf _{k \rightarrow \infty}} k P_{k}=0$, then $\left|f\left(c_{k}\right)\right| / r_{k+1} \rightarrow \infty$ and, setting

$$
A_{k+1}=\operatorname{ann}\left(0 ;\left(1+\varepsilon_{k+1}\right) r_{k+1},\left|f\left(c_{k}\right)\right|\right),
$$

we deduce from Theorem 1.2 that $\partial U_{k}$ is not uniformly perfect for $k \geq K$.

Suppose now that (1.10) holds. We show first that if $k$ is large, then

$$
\left\{z \in \mathbb{C}:\left(1-\frac{1}{k}\right) r_{k} \leq|z|<\left(1-\varepsilon_{k}\right) r_{k+1},\left|z-a_{k}\right| \geq \frac{1}{k} r_{k}\right\} \subseteq U_{k}
$$

but

$$
U_{k} \cap \bar{D}\left(a_{k}, \frac{\delta}{k} r_{k}\right)=\emptyset
$$

and

$$
U_{k} \cap\left[\frac{1}{2} a_{k},\left(1-\frac{\tau}{k}\right) a_{k}\right]=\emptyset
$$

for certain positive $\tau$ and $\delta$. Here $[u, v]$ is the line segment $\{u+t(v-u): 0 \leq t \leq 1\}$ connecting two points $u$ and $v$ in $\mathbb{C}$.

It follows from (4.6) and (4.7) that, in order to prove (4.13), it suffices to show that there exists $\varepsilon>0$ such that

$$
|f(z)| \geq(1+\varepsilon) r_{k+1} \quad \text { when }|z|=\left(1-\frac{1}{k}\right) r_{k}
$$

and

$$
|f(z)| \geq(1+\varepsilon) r_{k+1} \quad \text { when }\left|z-a_{k}\right|=\frac{1}{k} r_{k}
$$

for large $k$. Now (4.4) yields

$$
|f(z)| \geq(1-o(1))|C| \frac{(1-1 / k)^{k-1+N}}{2 k P_{k}} r_{k+1}=(1-o(1)) \frac{|C|}{2 e k P_{k}} r_{k+1}
$$


when $|z|=(1-1 / k) r_{k}$. Since $\lim \sup _{k \rightarrow \infty} k P_{k}<|C| /(2 e)$ by our assumption, (4.16) follows. Essentially the same argument also yields (4.17) and thus we obtain (4.13).

On the other hand, if $\left|z-a_{k}\right|=\delta r_{k} / k$, then $|z| \leq(1+\delta / k) r_{k}$, and we find by estimates similar to those before that

$$
|f(z)| \leq(1+o(1)) \frac{|C|(1+\delta / k)^{k-1+N} \delta}{2 k P_{k}} r_{k+1}=(1+o(1)) \frac{|C| e^{\delta} \delta}{2 k P_{k}} r_{k+1} .
$$

Since we assumed that $\lim _{\inf _{k \rightarrow \infty}} k P_{k}>0$, we see that if $\delta$ is sufficiently small, then

$$
|f(z)| \leq \frac{1}{2} r_{k+1}
$$

for large $k$. Thus $f(z) \in \widetilde{U}_{k}$. Since $\widetilde{U}_{k} \subset C\left(0, U_{k+1}\right)$ and thus

$$
\widetilde{U}_{k} \cap U_{k+1}=\widetilde{U}_{k} \cap f\left(U_{k}\right)=\emptyset,
$$

this yields (4.14).

Also, if $z=s a_{k}$ where $1 / 2 \leq s \leq(1-\tau / k)$, then

$$
|f(z)| \sim \frac{|C| s^{k-1+N}(1-s)}{2 P_{k}}
$$

by (4.4). The function $s \mapsto s^{k-1+N}(1-s)$ is increasing in the interval [1/2, $\left.(1-\tau / k)\right]$ when $\tau \geq 2$, and thus

$$
|f(z)| \leq(1+o(1)) \frac{|C|(1-\tau / k)^{k-1+N} \tau}{2 k P_{k}} r_{k+1} \sim \frac{|C| e^{-\tau} \tau}{2 k P_{k}} r_{k+1} .
$$

If $\tau$ is chosen sufficiently large, our assumption that $\lim _{\inf } f_{k \rightarrow \infty} k P_{k}>0$ implies (4.18) for large $k$. The same argument as above now yields (4.15).

We also note that, by construction,

$$
U_{k} \subseteq \operatorname{ann}\left(0 ;\left(1-\varepsilon_{k-1}\right) r_{k},\left(1+\varepsilon_{k+1}\right) r_{k+1}\right) \subseteq \operatorname{ann}\left(0 ; 1,2 r_{k+1}\right)
$$

for large $k$. We may assume that (4.13)-(4.15) and (4.19) hold when $k \geq K$.

In order to prove that $\partial U_{K}$ is uniformly perfect, we use Lemma 2.4. Let $\sigma_{K}$ be a curve in $U_{K}$ that is not null-homotopic. If $n\left(\sigma_{K}, 0\right) \neq 0$, then

$$
\operatorname{length}\left(\sigma_{K}, U_{K}\right) \geq \operatorname{length}\left(\sigma_{K}, \operatorname{ann}\left(0 ; 1,2 r_{K+1}\right)\right) \geq \frac{2 \pi^{2}}{\log \left(2 r_{K+1}\right)}
$$

by (4.19) and Lemma 2.5. Suppose that $n\left(\sigma_{K}, 0\right)=0$. Put $\sigma_{k}=f^{k-K}\left(\sigma_{K}\right)$ for $k>K$. By Theorem 1.1 (ii), we have $n\left(\sigma_{k}, 0\right) \neq 0$ for large $k$. Thus there exists $k \geq K$ such that $n\left(\sigma_{k}, 0\right)=0$ and $n\left(\sigma_{k+1}, 0\right) \neq 0$. It follows that $n\left(\sigma_{k}, a\right) \neq 0$ for some zero $a$ of $f$, and (4.13) implies that actually $n\left(\sigma_{k}, a_{k}\right) \neq 0$. Let

$$
V_{k}=\mathbb{C} \backslash\left(\bar{D}\left(a_{k}, \frac{\delta}{k} r_{k}\right) \cup\left[\frac{1}{2} a_{k},\left(1-\frac{\tau}{k}\right) a_{k}\right]\right) .
$$


Since $U_{k} \subseteq V_{k}$ by (4.14) and (4.15),

$$
\text { length }\left(\sigma_{K}, U_{K}\right) \geq \operatorname{length}\left(\sigma_{k}, U_{k}\right) \geq \operatorname{length}\left(\sigma_{k}, V_{k}\right) .
$$

We put

$$
T_{k}(z)=k\left(\frac{z-a_{k}}{a_{k}}\right) \quad \text { and } \quad W_{k}=T_{k}\left(V_{k}\right)=\mathbb{C} \mid\left(\bar{D}(0, \delta) \cup\left[-\frac{1}{2} k,-\tau\right]\right) .
$$

We may assume that $K \geq 4 \tau$. Take

$$
W=\mathbb{C} \backslash(\bar{D}(0, \delta) \cup[-2 \tau,-\tau]) ;
$$

then $W_{k} \subseteq W$ for $k \geq K$. Since $a_{k} / 2 \in C\left(0, U_{k}\right)$, we have $n\left(\sigma_{k}, a_{k} / 2\right)=0$. On the other hand, $n\left(\sigma_{k}, a_{k}\right) \neq 0$ and thus $\sigma_{k}$ separates $\left[a_{k} / 2,(1-\tau / k) a_{k}\right]$ and $\bar{D}\left(a_{k}, \delta r_{k} / k\right)$. Hence $T_{k}\left(\sigma_{k}\right)$ is a curve in $W$ that separates $\bar{D}(0, \delta)$ and $[-2 \tau,-\tau]$. Therefore

$$
\text { length }\left(\sigma_{k}, V_{k}\right)=\operatorname{length}\left(T_{k}\left(\sigma_{k}\right), W_{k}\right) \geq \operatorname{length}\left(T_{k}\left(\sigma_{k}\right), W\right) \geq c
$$

for some positive constant $c$. Combining (4.21) and (4.22), we obtain

$$
\text { length }\left(\sigma_{K}, U_{K}\right) \geq c \text {. }
$$

Now (4.20) and (4.23), together with Lemma 2.4, imply that $\partial U_{K}$ is uniformly perfect. Thus $\partial U_{k}$ is uniformly perfect when $k \geq K$ by Lemma 2.6.

Remark 4.1. As mentioned in the introduction, Kisaka and Shishikura [12] constructed an example of an entire function $f$ with a doubly connected wandering domain. In order to ensure that the wandering domains do not contain critical points, which has to be avoided by Lemma 2.2, they construct it in such a way that $f(0)=0$ and $f^{2}(c)=0$ for each critical point $c$ of $f$. Their construction uses quasiconformal surgery, but it turns out that the function obtained is of order zero and may be written in the form (1.8) with a sequence $\left(a_{k}\right)$ that tends to $\infty$ rapidly. The sequence $\left(c_{k}\right)$ of critical points again satisfies (4.9) and the construction is such that $f\left(c_{k}\right)=a_{k+1}$ and thus $f^{2}\left(c_{k}\right)=0$. It follows from the arguments in the above proof that

$$
a_{k+1}=f\left(c_{k}\right) \sim a_{k}^{N} \frac{C}{e k} \prod_{j=0}^{k-1} \frac{a_{k}}{a_{j}}
$$

as $k \rightarrow \infty$.

\section{Proof of Theorem 1.4}

It follows easily from Rolle's theorem that for a real polynomial with real zeros, each open interval on the real axis bounded by two adjacent zeros contains exactly 
one critical point, this critical point is simple, and there are no further critical points except for multiple zeros. Since $f$ is a locally uniform limit of polynomials with real zeros, the above result also holds for $f$. We thus find that, when $k \geq 0$, there exists a critical point $c_{k} \in\left(a_{k}, a_{k+1}\right)$, and except for 0 and one further critical point in the interval $\left(0, a_{0}\right)$, the $a_{k}$ and $c_{k}$ exhaust the critical points.

We will show that if $q_{0}$ is sufficiently large, then the following properties are satisfied for all $k \geq 0$ :

$$
\begin{aligned}
& f\left(\operatorname{ann}\left(4 a_{k}, a_{k+1} / 4\right)\right) \subseteq \operatorname{ann}\left(4 a_{k+1}, a_{k+2} / 4\right), \\
& f\left(\operatorname{ann}\left(4 a_{k}, \sqrt{a_{k+1}}\right)\right) \subseteq \operatorname{ann}\left(4 a_{k+1}, \sqrt{a_{k+2}}\right), \\
& \frac{q_{k}}{2 q_{k+1}} a_{k+1}<c_{k}<\frac{2 q_{k}}{q_{k+1}} a_{k+1},
\end{aligned}
$$

and

$$
\sqrt{a_{k+2}}<f\left(c_{k}\right)<\frac{1}{4} a_{k+2} .
$$

Suppose that (5.1)-(5.4) hold. Then $f$ has a wandering domain $U_{0}$ that contains $\operatorname{ann}\left(4 a_{0}, a_{1} / 4\right)$.

We consider the annulus $X_{k}=D\left(0, \sqrt{a_{k+1}}\right) \backslash C\left(0, U_{k}\right)$. If $k \geq 1$, then $X_{k}$ contains no critical values by (5.4), and thus the components of $f^{-1}\left(X_{k}\right)$ are also annuli. In particular, this holds for the component $Y_{k-1}$ of $f^{-1}\left(X_{k}\right)$ whose boundary intersects $C\left(0, U_{k-1}\right)$. By (5.2), we have $Y_{k-1} \supseteq X_{k-1}$ and thus $f\left(X_{k-1}\right) \subseteq f\left(Y_{k-1}\right)=X_{k}$. It follows that $X_{k} \subseteq F(f)$ and thus $X_{k} \subseteq U_{k}$ for all $k \geq 0$. This implies that

$$
a_{j} \in C\left(0, U_{k}\right)
$$

when $0 \leq j \leq k$. We also note that

$$
U_{k} \subseteq \operatorname{ann}\left(a_{k} / 4,4 a_{k+1}\right) \subseteq \operatorname{ann}\left(1,4 a_{k+1}\right)
$$

when $k \geq 1$ and

$$
U_{0} \subseteq \operatorname{ann}\left(\delta, 4 a_{1}\right)
$$

for some positive $\delta$, since 0 is a superattracting fixed point.

As in the proof of Theorem 1.3, we use Lemma 2.4 to show that $\partial U_{0}$ is uniformly perfect. Let $\sigma_{0}$ be a Jordan curve in $U_{0}$ that is not null-homotopic. If $n\left(\sigma_{0}, 0\right) \neq 0$, then

$$
\operatorname{length}\left(\sigma_{0}, U_{0}\right) \geq \frac{2 \pi^{2}}{\log \left(4 a_{1} / \delta\right)}
$$

by (5.7) and Lemma 2.5.

We now assume that $n\left(\sigma_{0}, 0\right)=0$. Put $\sigma_{k}=f^{k}\left(\sigma_{0}\right)$. By Theorem 1.1 (ii), we have $n\left(\sigma_{k}, 0\right) \neq 0$ for large $k$, and thus there exists $k \geq 1$ such that $n\left(\sigma_{k}, 0\right) \neq 0$ while $n\left(\sigma_{k-1}, 0\right)=0$. It follows that $n\left(\sigma_{k-1}, a\right) \neq 0$ for some zero $a$ of $f$. Using (5.5), we see 
that $n\left(\sigma_{k-1}, a_{k}\right) \neq 0$ while $n\left(\sigma_{k-1}, a_{j}\right)=0$ for $j \neq k$. Since $a_{k}$ is a zero of multiplicity $q_{k}$, this implies that

$$
n\left(\sigma_{k}, 0\right)=n\left(f\left(\sigma_{k-1}\right), 0\right)=q_{k} n\left(\sigma_{k-1}, a_{k}\right)
$$

In particular, $\left|n\left(\sigma_{k}, 0\right)\right| \geq q_{k}$. Combining this with (5.6) and Lemma 2.5, we obtain

$$
\operatorname{length}\left(\sigma_{k}, U_{k}\right) \geq \operatorname{length}\left(\sigma_{k}, \operatorname{ann}\left(1,4 a_{k+1}\right)\right) \geq \frac{2 \pi^{2} q_{k}}{\log \left(4 a_{k+1}\right)}=\frac{2 \pi^{2} q_{k}}{q_{k}+\log 4} \geq \pi^{2} .
$$

On the other hand, length $\left(\sigma_{k}, U_{k}\right) \leq \operatorname{length}\left(\sigma_{0}, U_{0}\right)$ and thus

$$
\text { length }\left(\sigma_{0}, U_{0}\right) \geq \pi^{2}
$$

in this case. Thus, for each Jordan curve $\sigma_{0}$ in $U_{0}$ that is not null-homotopic, (5.8) or (5.9) holds. Lemma 2.4 now implies that $\partial U_{0}$ is uniformly perfect.

Next, $l_{k}=1$ since $f\left(c_{j}\right) \in U_{j+1} \subset C\left(0, U_{k+1}\right)$ when $j<k$ by (5.4), so that $c_{k}$ is the only critical point of $f$ in $\widetilde{U}_{k}$ that is not mapped into $C\left(0, U_{k+1}\right)$. Further,

$$
n_{k}=2+\sum_{j=0}^{k+1} q_{j} \quad \text { and } \quad m_{k}=2+\sum_{j=0}^{k} q_{j}
$$

by (5.5). Thus (1.4) is satisfied. Note that $n_{k}>m_{k}$.

In order to prove (1.12), we note that by (5.4) the annulus

$$
A_{k+1}=\operatorname{ann}\left(4 a_{k+1}, \sqrt{a_{k+2}}\right)
$$

separates $f\left(c_{k}\right)$ and $C\left(0, U_{k+1}\right)$. Now

$$
\frac{\bmod \left(A_{k+1}\right)}{n_{k}-m_{k}}=\frac{\log \left(\sqrt{a_{k+2}} / 4 a_{k+1}\right)}{2 \pi q_{k+1}}=\frac{q_{k+1} / 2-\log 4-q_{k}}{2 \pi q_{k+1}} \rightarrow \frac{1}{4 \pi}
$$

as $k \rightarrow \infty$, and this proves (1.12).

It remains to prove (5.1)-(5.4). To do this, we note first that $q_{k+1} \geq 3 q_{0} q_{k} / 2$ and thus $q_{k} \geq\left(3 q_{0} / 2\right)^{k-j} q_{j}$ when $k>j$. Thus

$$
\sum_{j=0}^{k-1} q_{j} \leq q_{k-1} \sum_{j=0}^{k-1}\left(\frac{2}{3 q_{0}}\right)^{j}
$$

which implies that, given a small positive $\varepsilon$, we can arrange that

$$
\sum_{j=0}^{k-1} q_{j} \leq(1+\varepsilon) q_{k-1}
$$

by choosing $q_{0}$ large enough. In particular, $q_{k-2} \leq \varepsilon q_{k-1}$. 
We also note that the sequence $\left(a_{k}\right)$ tends to $\infty$ very rapidly, and using this it is not difficult to see that we can ensure that

$$
\prod_{j=k+1}^{\infty}\left(1+\frac{4 a_{k}}{a_{j}}\right)^{q_{j}} \leq 2 \text { and } \prod_{j=k+1}^{\infty}\left(1-\frac{4 a_{k}}{a_{j}}\right)^{q_{j}} \geq \frac{1}{2}
$$

for all $k$ by choosing $q_{0}$ large. We can also arrange that

$$
1+\frac{a_{k}}{4 a_{j}} \leq a_{k} \quad \text { and } \quad \frac{a_{k}}{4 a_{j}}-1 \geq \frac{a_{k}}{5 a_{j}}
$$

when $0 \leq j<k$. If $k \geq 1$ and $|z|=a_{k} / 4$, then

$$
\begin{aligned}
|f(z)| & \leq \frac{1}{16} a_{k}^{2} \prod_{j=0}^{k-1}\left(1+\frac{a_{k}}{4 a_{j}}\right)^{q_{j}}\left(\frac{5}{4}\right)^{q_{k}} \prod_{j=k+1}^{\infty}\left(1+\frac{a_{k}}{4 a_{j}}\right)^{q_{j}} \\
& \leq \frac{1}{8} a_{k}^{2} \prod_{j=0}^{k-1} a_{k}^{q_{j}}\left(\frac{5}{4}\right)^{q_{k}} \\
& =\frac{1}{8} \exp \left(2 q_{k-1}+q_{k-1} \sum_{j=0}^{k-1} q_{j}+q_{k} \log \frac{5}{4}\right) \\
& \leq \frac{1}{8} \exp \left(2 \varepsilon q_{k}+(1+\varepsilon) q_{k-1}^{2}+q_{k} \log \frac{5}{4}\right) \\
& =\frac{1}{8} \exp \left(\left(2 \varepsilon+(1+\varepsilon) \frac{2}{3}+\log \frac{5}{4}\right) q_{k}\right)
\end{aligned}
$$

by (1.11) and (5.10)-(5.12). Choosing $\varepsilon$ small, which may be done by choosing $q_{0}$ large, we obtain

$$
|f(z)| \leq \frac{1}{8} \exp q_{k}=\frac{1}{8} a_{k+1}
$$

when $|z|=a_{k} / 4$. Further, when $k \geq 1$ and $|z|=a_{k} / 4$,

$$
\begin{aligned}
|f(z)| & \geq \frac{1}{16} a_{k}^{2} \prod_{j=0}^{k-1}\left(\frac{a_{k}}{4 a_{j}}-1\right)^{q_{j}}\left(\frac{3}{4}\right)^{q_{k}} \prod_{j=k+1}^{\infty}\left(1-\frac{a_{k}}{4 a_{j}}\right)^{q_{j}} \\
& \geq \frac{1}{32} a_{k}^{2} \prod_{j=1}^{k-1}\left(\frac{a_{k}}{5 a_{j}}\right)^{q_{j}}\left(\frac{3}{4}\right)^{q_{k}} \\
& =\frac{a_{k}}{32} \exp \left(q_{k-1}+\sum_{j=1}^{k-1} q_{j}\left(q_{k-1}-q_{j-1}-\log 5\right)+q_{k} \log \frac{3}{4}\right),
\end{aligned}
$$

by (5.11) and (5.12). As before, we see that

$$
q_{k-1}+\sum_{j=1}^{k-1} q_{j}\left(q_{k-1}-q_{j-1}-\log 5\right) \geq q_{k}(1-\varepsilon) \frac{2}{3}
$$


for large $q_{0}$, and since we may choose $\varepsilon$ such that $(1-\varepsilon) 2 / 3+\log (3 / 4)>0$, we see that

$$
|f(z)| \geq 4 a_{k}
$$

when $|z|=a_{k} / 4$. If $k \geq 1$ and $|z|=4 a_{k}$, then

$$
|f(z)| \geq 16 a_{k}^{2} \prod_{j=0}^{k-1}\left(\frac{4 a_{k}}{a_{j}}-1\right)^{q_{j}} 3^{q_{k}} \prod_{j=k+1}^{\infty}\left(1-\frac{4 a_{k}}{a_{j}}\right)^{q_{j}}
$$

and thus

$$
|f(z)| \geq 8 \cdot 3^{q_{k}} \geq 4 a_{k+1}
$$

when $|z|=4 a_{k}$ by (5.11). If $|z|=4 a_{0}$, then

$$
|f(z)| \geq 16 a_{0}^{2} 3^{q_{0}} \prod_{j=1}^{\infty}\left(1-\frac{4 a_{0}}{a_{j}}\right)^{q_{j}} \geq 8 a_{0}^{2} 3^{q_{0}}=8 a_{1} 3^{q_{0}} \geq 4 a_{1},
$$

so that (5.15) also holds if $k=0$. By (5.13), $|f(z)| \leq a_{k+2} / 4$ when $|z|=a_{k+1} / 4$. Since $a_{k+1} / 4>4 a_{k}$ for large $q_{0}$, we see also that

$$
|f(z)| \leq \frac{1}{4} a_{k+2} \quad \text { when }|z|=4 a_{k} .
$$

Now (5.1) follows from (5.13)-(5.16).

When $k \geq 1$ and $|z|=\sqrt{a_{k}}$, we see as before that

$$
\begin{aligned}
|f(z)|^{2} & \leq a_{k}^{2} \prod_{j=0}^{k-1}\left(1+\frac{\sqrt{a_{k}}}{a_{j}}\right)^{2 q_{j}}\left(1+\frac{1}{\sqrt{a_{k}}}\right)^{2 q_{k}} 2 \\
& \leq 2 a_{k}^{2} \prod_{j=0}^{k-1} a_{k}^{q_{j}}(1+\varepsilon)^{2 q_{k}} \\
& =\exp \left(\log 2+2 q_{k-1}+q_{k-1} \sum_{j=0}^{k-1} q_{j}+2 q_{k} \log (1+\varepsilon)\right) \\
& \leq \exp \left(\left((1+\varepsilon) \frac{2}{3}+3 \varepsilon+2 \log (1+\varepsilon)\right) q_{k}\right) \\
& \leq a_{k+1}
\end{aligned}
$$

for large $q_{0}$ and small $\varepsilon$. This yields (5.2).

The location of the critical points could be determined by Rouchés theorem as in the proof of Theorem 1.3. Alternatively, to prove (5.3) it suffices to show that

$$
f^{\prime}\left(x_{k}\right)>0 \text { and } f^{\prime}\left(y_{k}\right)<0,
$$


where

$$
x_{k}=\frac{q_{k}}{2 q_{k+1}} a_{k+1} \quad \text { and } \quad y_{k}=\frac{2 q_{k}}{q_{k+1}} a_{k+1} .
$$

Since $f(x) \geq 0$ for all $x \in \mathbb{R}$, it suffices to prove these inequalities with $f^{\prime}$ replaced by $f^{\prime} / f$. Now

$$
\frac{f^{\prime}(z)}{f(z)}=\frac{2}{z}+\sum_{j=0}^{\infty} \frac{q_{j}}{z-a_{j}}
$$

Thus

$$
\frac{f^{\prime}\left(x_{k}\right)}{f\left(x_{k}\right)}>\sum_{j=k}^{\infty} \frac{q_{j}}{x_{k}-a_{j}}=\frac{q_{k}}{x_{k}-a_{k}}-\frac{q_{k+1}}{a_{k+1}-x_{k}}-\sum_{j=k+2}^{\infty} \frac{q_{j}}{a_{j}-x_{k}} .
$$

For large $q_{0}$,

$$
\frac{q_{k}}{x_{k}-a_{k}}=\frac{2 q_{k} q_{k+1}}{q_{k} a_{k+1}-2 q_{k+1} a_{k}} \geq \frac{7}{4} \frac{q_{k+1}}{a_{k+1}},
$$

while

$$
\frac{q_{k+1}}{a_{k+1}-x_{k}}=\frac{2 q_{k+1}^{2}}{2 q_{k+1} a_{k+1}-q_{k} a_{k+1}} \leq \frac{5}{4} \frac{q_{k+1}}{a_{k+1}},
$$

and

$$
\sum_{j=k+2}^{\infty} \frac{q_{j}}{a_{j}-x_{k}} \leq 2 \sum_{j=k+2}^{\infty} \frac{q_{j}}{a_{j}} \leq \frac{1}{4} \frac{q_{k+1}}{a_{k+1}} .
$$

These last inequalities imply that $f^{\prime}\left(x_{k}\right)>0$. Similarly, for large $q_{0}$,

$$
\begin{aligned}
\frac{f^{\prime}\left(y_{k}\right)}{f\left(y_{k}\right)} & \leq \frac{2}{y_{k}}+\sum_{j=0}^{k} \frac{q_{j}}{y_{k}-a_{j}}-\frac{q_{k+1}}{a_{k+1}-y_{k}} \\
& =\frac{q_{k+1}}{q_{k} a_{k+1}}+\sum_{j=0}^{k} \frac{q_{j} q_{k+1}}{2 q_{k} a_{k+1}-q_{k+1} a_{j}}-\frac{q_{k+1}^{2}}{q_{k+1} a_{k+1}-2 q_{k} a_{k+1}} \\
& \leq \frac{q_{k+1}}{q_{k} a_{k+1}}+\frac{5}{8} \sum_{j=0}^{k} \frac{q_{j} q_{k+1}}{q_{k} a_{k+1}}-\frac{7}{8} \frac{q_{k+1}}{a_{k+1}} \\
& =\frac{q_{k+1}}{a_{k+1}}\left(\frac{1}{q_{k}}+\frac{5}{8} \frac{1}{q_{k}} \sum_{j=0}^{k} q_{j}-\frac{7}{8}\right) \\
& <0,
\end{aligned}
$$

and thus $f^{\prime}\left(y_{k}\right)<0$. This completes the proof of (5.3). 
The right-hand inequality of (5.4) follows from (5.1) and (5.3). To prove the other inequality, we note that

$$
\begin{aligned}
f\left(c_{k}\right) & \geq f\left(x_{k}\right) \\
& =x_{k}^{2} \prod_{j=0}^{k}\left(\frac{x_{k}}{a_{j}}-1\right)^{q_{j}}\left(1-\frac{x_{k}}{a_{k+1}}\right)^{q_{k+1}} \prod_{j=k+2}^{\infty}\left(1-\frac{x_{k}}{a_{j}}\right)^{q_{j}} \\
& \geq x_{k}^{2}\left(\frac{x_{k}}{a_{k}}-1\right)^{q_{k}}\left(1-\frac{x_{k}}{a_{k+1}}\right)^{q_{k+1}} \frac{1}{2} \\
& \geq\left(\frac{x_{k}}{2 a_{k}}\right)^{q_{k}}\left(1-\frac{x_{k}}{a_{k+1}}\right)^{q_{k+1}} \\
& =\left(\frac{q_{k} a_{k+1}}{4 q_{k+1} a_{k}}\right)^{q_{k}}\left(1-\frac{q_{k}}{2 q_{k+1}}\right)^{q_{k+1}} \\
& \geq\left(\frac{q_{k} a_{k+1}}{4 q_{k+1} a_{k}}\right)^{q_{k}} \exp \left(-q_{k-1}\right)
\end{aligned}
$$

for large $q_{0}$. Noting that

$$
q_{k}=\frac{3}{2} q_{k-1}^{2}=\frac{3}{2}\left(\log a_{k}\right)^{2}
$$

and

$$
a_{k}=\exp q_{k-1}=\exp \sqrt{\frac{2}{3} q_{k}} \leq \exp \sqrt{q_{k}}=\exp \sqrt{\log a_{k+1}},
$$

we see that, given any positive $\delta$, we can ensure that

$$
q_{k} \leq a_{k}^{\delta} \quad \text { and } \quad a_{k} \leq a_{k+1}^{\delta}
$$

by choosing $q_{0}$ large. Thus, given any positive $\varepsilon$, we deduce from (5.17) that

$$
f\left(c_{k}\right) \geq a_{k+1}^{(1-\varepsilon) q_{k}} \exp \left(-q_{k}\right)=\exp \left((1-\varepsilon) q_{k}^{2}-q_{k}\right) \geq \exp \left((1-2 \varepsilon) \frac{2}{3} q_{k+1}\right)
$$

for large $q_{0}$. If $\varepsilon$ is small enough,

$$
f\left(c_{k}\right) \geq \exp \left(\frac{1}{2} q_{k+1}\right)=\sqrt{a_{k+2}} .
$$

This completes the proof of (5.4) and thus the proof of Theorem 1.4.

Remark 5.1. Let $f$ be a transcendental entire function with a multiply connected wandering domain $U_{0}$ and put $U_{k}=f^{k}\left(U_{0}\right)$, as before. By Theorem 1.1 (iii), the $U_{k}$ are all bounded and thus $\partial U_{k} \cap C\left(\infty, U_{k}\right)$ is connected. We call $\partial U_{k} \cap C\left(\infty, U_{k}\right)$ the outer boundary of $U_{k}$ and denote it by $\partial_{\infty} U_{k}$. By Theorem 1.1 (ii), we have $0 \in C\left(0, U_{k}\right)$ for large $k$, and for such $k$ we call $\partial U_{k} \cap C\left(0, U_{k}\right)$ the inner boundary of $U_{k}$, and denote it by $\partial_{0} U_{k}$. It is not difficult to see when $U_{k}$ is infinitely connected, then the components 
of $\partial U_{k}$ must cluster at the inner or outer boundary (or both). However, as we will explain below, it may happen that they cluster at only one of these.

In the proof of Theorem 1.4, we have shown that, with the terminology used there, $X_{k}=D\left(0, \sqrt{a_{k+1}}\right) \backslash C\left(0, U_{k}\right) \subseteq U_{k}$. This implies that the components of $\partial U_{k}$ do not cluster at the inner boundary $\partial_{0} U_{k}$ : it is an isolated part of the boundary in the sense that $\operatorname{dist}\left(\partial_{0} U_{k}, \partial U_{k} \backslash \partial_{0} U_{k}\right)>0$.

On the other hand, if $f$ is as in Theorem 1.3, with $\left(P_{k}\right)$ chosen such that

$$
\limsup _{k \rightarrow \infty} k P_{k}<\frac{|C|}{2 e},
$$

then the outer boundary of $U_{k}$ is an isolated part of the boundary. In fact, the proof of Theorem 1.3 shows that (5.18) implies (4.12) for all large $k$. We deduce that $S_{k+1}$, defined to be $\widetilde{U}_{k+1} \backslash \bar{D}\left(0,2^{k} r_{k+1}\right)$, contains no critical values. Thus the components of $f^{-1}\left(S_{k+1}\right)$ are doubly connected. Proceeding as in the proof of (4.5), we can show that $|f(z)| \geq 2^{k} r_{k+1}$ when $|z|=2^{k-1} r_{k}$. This implies that there exists a component $T_{k}$ of $f^{-1}\left(S_{k+1}\right)$ that contains $S_{k}$. It follows that $f\left(S_{k}\right) \subseteq f\left(T_{k}\right)=S_{k+1}$, and this implies that $S_{k} \subseteq F(f)$. Thus the outer boundary $\partial_{\infty} U_{k}$ of $U_{k}$ is isolated.

Similar reasoning shows that if

$$
\liminf _{k \rightarrow \infty} k P_{k}>\frac{|C|}{2 e},
$$

then $\partial_{0} U_{k}$ is isolated, and if

$$
\liminf _{k \rightarrow \infty} k P_{k}<\frac{|C|}{2 e}<\limsup _{k \rightarrow \infty} k P_{k},
$$

then neither $\partial_{0} U_{k}$ nor $\partial_{\infty} U_{k}$ is isolated.

These examples illustrate a result in [9, Theorem 8.1], where it is shown that the inner or outer boundary is isolated under a suitable hypothesis on the location of the critical points.

\section{Baker's example of an infinitely connected Fatou component}

In Baker's example (1.3), the constant $C$ and the sequence $\left(r_{k}\right)$ are chosen as follows. First take $C \in\left(0,1 /\left(4 e^{2}\right)\right)$ and $r_{1}>1$. Then choose $k_{0}$ such that $2^{k_{0}-1} C>2 r_{1}$. Finally choose $\left(r_{k}\right)$ such that $r_{k+1} \geq 2 r_{k}$ when $1 \leq k<k_{0}$, and put

$$
r_{k+1}=C^{2} \prod_{j=1}^{k}\left(1+\frac{r_{k}}{r_{j}}\right)^{2}
$$

when $k \geq k_{0}$.

Baker showed that, if

$$
s_{k}=\frac{k+1}{k+2} r_{k+1} \quad \text { and } \quad B_{k}=\operatorname{ann}\left(r_{k}^{2}, s_{k}\right)
$$


then $f\left(B_{k}\right) \subseteq B_{k+1}$ for large $k$ and thus $B_{k} \subseteq U_{k}$ for some multiply connected wandering domain $U_{k}$. To prove that $U_{k}$ is infinitely connected, Baker found a critical point

$$
c_{k+1} \in\left(-s_{k},-r_{k}^{2}\right) \subset B_{k}
$$

for large $k$. The infinite connectivity then follows from Lemma 2.2.

Analogously to (4.9), we now find that

$$
c_{k}=-\left(1-\frac{1}{k+\delta_{k}}\right) r_{k}
$$

where $\delta_{k} \rightarrow 0$, and instead of (4.11), we now obtain

$$
\left|f\left(c_{k}\right)\right| \sim \frac{C^{2}}{e k} \prod_{j=1}^{k-1}\left(1+\frac{r_{k}}{r_{j}}\right)^{2}=\frac{1}{4 e k} r_{k+1} .
$$

Similar to (4.1), we also have $r_{k+1} \geq 2^{k} r_{k}^{2}$. Hence $\left|f\left(c_{k}\right)\right| / r_{k}^{2} \rightarrow \infty$. As $c_{k+1}$ is the only critical point in $U_{k}$, it now follows from Theorem 1.2, applied with $A_{k+1}$ taken to be $\operatorname{ann}\left(r_{k+1}^{2},\left|f\left(c_{k+1}\right)\right|\right) \subseteq U_{k+1}$, that $\partial U_{k}$ is not uniformly perfect.

\section{Acknowledgements}

We thank the referee for many helpful comments and Alexandre Eremenko for a discussion of his unpublished preprint and references [11, 13].

\section{References}

[1] I. N. Baker, 'Multiply connected domains of normality in iteration theory', Math. Z. 81 (1963), 206-214.

[2] I. N. Baker, 'The domains of normality of an entire function', Ann. Acad. Sci. Fenn. Ser. A I Math. 1 (1975), 277-283.

[3] I. N. Baker, 'An entire function which has wandering domains', J. Aust. Math. Soc. (Ser. A) 22 (1976), 173-176.

[4] I. N. Baker, 'Wandering domains in the iteration of entire functions', Proc. Lond. Math. Soc. (3) 49 (1984), 563-576.

[5] I. N. Baker, 'Infinite limits in the iteration of entire functions', Ergodic Theory Dynam. Systems $\mathbf{8}$ (1988), 503-507.

[6] A. F. Beardon and Ch. Pommerenke, 'The Poincaré metric of plane domains', J. Lond. Math. Soc. 18 (1978), 475-483.

[7] W. Bergweiler, 'Iteration of meromorphic functions', Bull. Amer. Math. Soc. (N.S.) 29 (1993), 151-188.

[8] W. Bergweiler, 'Connectivity of Fatou components', Oberwolfach Rep. 6 (2009), 2946-2948.

[9] W. Bergweiler, P. J. Rippon and G. M. Stallard, 'Multiply connected wandering domains of entire functions', Preprint, arXiv:1109.1794.

[10] A. Eremenko, unpublished manuscript, http://www.math.purdue.edu/ eremenko/dvi/ups.pdf.

[11] A. Hinkkanen, 'Julia sets of rational functions are uniformly perfect', Math. Proc. Cambridge Philos. Soc. 113 (1993), 543-559. 
[12] M. Kisaka and M. Shishikura, 'On multiply connected wandering domains of entire functions', in: Transcendental Dynamics and Complex Analysis, LMS Lecture Note Series, 348 (eds. P. J. Rippon and G. M. Stallard) (Cambridge University Press, Cambridge, 2008), pp. 217-250.

[13] R. Mañé and L. F. da Rocha, 'Julia sets are uniformly perfect', Proc. Amer. Math. Soc. 116 (1992), 251-257.

[14] C. T. McMullen, Complex Dynamics and Renormalization, Annals of Mathematics Studies, 135 (Princeton University Press, Princeton, NJ, 1994).

[15] Ch. Pommerenke, 'Uniformly perfect sets and the Poincaré metric', Arch. Math. (Basel) 32 (1979), 192-199.

[16] D. Schleicher, 'Dynamics of entire functions', in: Holomorphic Dynamical Systems, Lecture Notes in Mathematics, 1998 (Springer, Heidelberg-Dordrecht-London-New York, 2010), pp. 295-339.

[17] N. Steinmetz, Rational Iteration (Walter de Gruyter, Berlin, 1993).

[18] T. Sugawa, 'Various domain constants related to uniform perfectness', Complex Variables Theory Appl. 36 (1998), 311-345.

[19] D. Sullivan, 'Quasiconformal homeomorphisms and dynamics I. Solution of the Fatou-Julia problem on wandering domains', Ann. of Math. (2) 122 (1985), 401-418.

[20] H. Töpfer, 'Über die Iteration der ganzen transzendenten Funktionen, insbesondere von $\sin z$ und cos z', Math. Ann. 117 (1939), 65-84.

[21] J. H. Zheng, 'On uniformly perfect boundaries of stable domains in iteration of meromorphic functions', Bull. Lond. Math. Soc. 32 (2000), 439-446.

[22] J. H. Zheng, 'Uniformly perfect sets and distortion of holomorphic functions', Nagoya Math. J. 164 (2001), 17-33.

[23] J. H. Zheng, 'On multiply-connected Fatou components in iteration of meromorphic functions', J. Math. Anal. Appl. 313 (2006), 24-37.

WALTER BERGWEILER, Mathematisches Seminar, Christian-Albrechts-Universität zu Kiel, Ludewig-Meyn-Str. 4, D-24098 Kiel, Germany

e-mail: bergweiler@math.uni-kiel.de

JIAN-HUA ZHENG, Department of Mathematical Sciences, Tsinghua University, 100084, Beijing, PR China

e-mail: jzheng@math.tsinghua.edu.cn 